\title{
On Approximating the Smallest Enclosing Bregman Balls
}

\author{
Frank Nielsen \\ Sony Computer Science Laboratories Inc. \\ Tokyo, Japan \\ Frank.Nielsen@acm.org
}

\author{
Richard Nock \\ University of Antilles-Guyane \\ Martinique, France \\ Richard.Nock@martinique.univ-ag.fr
}

\begin{abstract}
We present a generalization of Bădoiu and Clarkson's algorithm [3] for computing a $(1+\epsilon)$-approximation of the smallest enclosing ball of a point set equipped with a Bregman divergence as a distortion measure.
\end{abstract}

\section{Categories and Subject Descriptors}

I.3.5 [Computer Graphics]: Computational Geometry and Object Modeling - Geometric algorithms, languages, and systems

General Terms:

Algorithms, Theory

Keywords

Minimum enclosing ball, core-set, Bregman divergence

\section{INTRODUCTION}

Given a set $\mathcal{S}=\left\{\mathbf{s}_{i}\right\}_{i=1}^{n}$ of $d$-dimensional points, we are interested in finding a center point $\mathbf{c}^{*}$ of $\mathcal{S}$, where the "distance" measure $d(\cdot, \cdot)$ between any two points of $\mathcal{S}$, is a divergence (also called distortion ${ }^{1}$ ). Two optimization criteria may be considered for finding such a center: MiNAvG which minimizes the average divergence or MinMax which minimizes the maximal divergence $\left(\mathbf{c}^{*}=\operatorname{argmin}_{\mathbf{c}} d(\mathbf{c}, \mathcal{S})\right.$, where $d(\mathbf{c}, \mathcal{S})$ denote the divergence from $\mathbf{c}$ to the furthest point of $\left.\mathcal{S}: d(\mathbf{c}, \mathcal{S})=\max _{i} d\left(\mathbf{c}, \mathbf{s}_{i}\right)\right)$. These problems have been widely studied in computational geometry (1-center problem), computational statistics (1-point estimator), and machine learning (1-class classification). It is known that for the squared Euclidean distance $\left(L_{2}^{2}\right)$ the centroid $\frac{1}{n} \sum_{i=1}^{n} \mathbf{s}_{i}$ is the $\operatorname{MinAvg}\left(L_{2}^{2}\right)$ center [2]. For the Euclidean distance $L_{2}$, the circumcenter of $\mathcal{S}$ is the $\operatorname{MinMax}\left(L_{2}\right)$ center, and the Fermat-Weber point is the $\operatorname{Min} \operatorname{Avg}\left(L_{2}\right)$ center (see Figure 1). Finding the circumcenter of the unique smallest enclosing ball of $\mathcal{S}$ is weakly polynomial, and can be solved efficiently either numerically using second-order cone programming (SOCP), or combinatorially using a recent ball deflating heuristic, up to dimension 1000 and more [1].

\section{BREGMAN DIVERGENCES}

In computational machine learning, the $L_{2}$ geometric distance seldomly reflects the distance between two $d$-dimensional

\footnotetext{
*http://www.csl.sony.co.jp/person/nielsen/BregmanBall

${ }^{1}$ Symmetry and triangle inequality properties may not hold.
}

Copyright is held by the author/owner(s).

SCG'06, June 5-7, 2006, Sedona, Arizona, USA.

ACM 1-59593-340-9/06/0006.

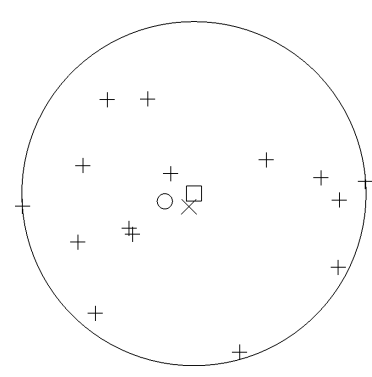

Figure 1: Three centers of a point set: centroid $(\times)$, circumcenter $(\square)$ and Fermat-Weber point (o).

points. A more general distance framework, known as Bregman divergences, is rather used. Bregman divergences $D_{F}$ are parameterized families of distortions defined on a convex domain $\mathcal{X} \subseteq \mathbb{R}^{d}$ for strictly convex and differentiable functions $F$ on $\operatorname{int}(\mathcal{X})$ (Figure 2) as $D_{F}(\mathbf{p}, \mathbf{q})=F(\mathbf{p})-$ $F(\mathbf{q})-\left\langle\mathbf{p}-\mathbf{q}, \boldsymbol{\nabla}_{F}(\mathbf{q})\right\rangle$, where $\boldsymbol{\nabla}_{F}$ denotes the gradient operator, and $\langle\cdot, \cdot\rangle$ the inner product (dot product). Informally speaking, a Bregman divergence $D_{F}$ is the tail of a Taylor expansion of $F$. Bregman divergences include the squared Euclidean distance $D_{F}(\mathbf{p}, \mathbf{q})=\|\mathbf{p}-\mathbf{q}\|^{2}(F(\mathbf{x})=$ $\|\mathbf{x}\|^{2}$ ), the Kullback-Leibler divergence (also known as the Information divergence) $D_{F}(\mathbf{p}, \mathbf{q})=\sum_{i=1}^{d} p_{i} \log \frac{p_{i}}{q_{i}}(F(\mathbf{x})=$ $\sum_{i=1}^{d} x_{i} \log x_{i}$ the negative entropy defined on the $d$-simplex), and the Itakura-Saito divergence $D_{F}(\mathbf{p}, \mathbf{q})=\sum_{i=1}^{d}\left(\frac{p_{i}}{q_{i}}-\right.$ $\left.\log \frac{p_{i}}{q_{i}}-1\right)\left(F(\mathbf{x})=-\sum_{i=1}^{d} \log x_{i}\right.$ defined on $\left.\mathbb{R}_{+}^{d}\right)$. Bregman divergences define two families of Bregman balls: $\mathcal{B}_{\mathbf{c}, r}=$ $\left\{\mathbf{x} \in \mathcal{X}: D_{F}(\mathbf{c}, \mathbf{x}) \leq r\right\}$ and $\mathcal{B}_{\mathbf{c}, r}^{\prime}=\left\{\mathbf{x} \in \mathcal{X}: D_{F}(\mathbf{x}, \mathbf{c}) \leq r\right\}$, that are not necessarily convex nor identical (Figure 3 ). For a point set $\mathcal{S}$, the two smallest enclosing balls $\mathcal{B}^{*}(\mathcal{S})$ and $\mathcal{B}^{\prime *}(\mathcal{S})$ have been shown to be unique [4]. Bregman balls have many important applications in machine learning. For example, finding the minimum enclosing Itakura-Saito ball

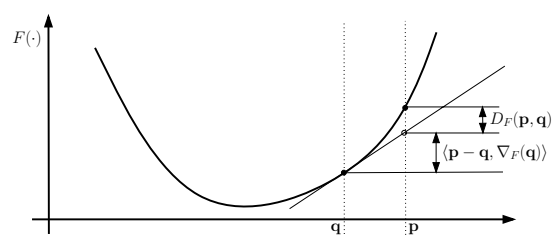

Figure 2: Visualizing the convex function $F$ and its associated Bregman divergence $D_{F}(\cdot, \cdot)$. 


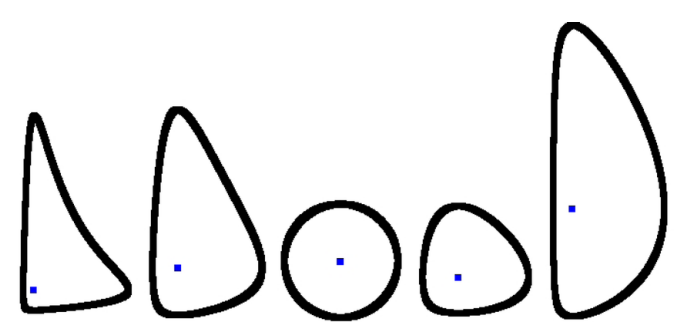

Figure 3: Examples of Itakura-Saito $(\times 2)$, squared Euclidean $(\times 1)$ and Kullback-Leibler $(\times 2)$ balls.

is used to find the closest signal to a set of given signals in speech recognition [4].

\section{APPROXIMATION ALGORITHMS}

Bădoiu and Clarkson [3] introduced the notion of coresets for balls. An $\epsilon$-core-set $\mathcal{C}$ for the MinMax ball of $\mathcal{S}$ is a subset $\mathcal{C} \subseteq \mathcal{S}$ such that the circumcenter c of the MinMax ball of $\mathcal{C}$ is such that $d(\mathbf{c}, \mathcal{S}) \leq(1+\epsilon) r^{*}$, where $r^{*}$ is the radius of the smallest enclosing ball of $\mathcal{S}$. They show that core-set sizes are independent of the dimension, and of size bounded by $\frac{2}{\epsilon}$. Further, they described a simple guaranteed $O\left(\frac{d n}{\epsilon^{2}}\right)$-time $(1+\epsilon)$-approximation algorithm. Applying this algorithm to "skewed" divergences does not make sense and yield poor results [4]. In [4], we generalize their approximation algorithm to arbitrary Bregman divergences: BBC. Choose at random $\mathbf{c} \in \mathcal{S}$

for $t=1,2, \ldots, T$ do

$$
\begin{aligned}
& \mathbf{s} \leftarrow \arg \max _{\mathbf{s}^{\prime} \in \mathcal{S}} D_{F}\left(\mathbf{c}, \mathbf{s}^{\prime}\right) \\
& \mathbf{c} \leftarrow \nabla_{F}^{-1}\left(\frac{t}{t+1} \nabla_{F}(\mathbf{c})+\frac{1}{t+1} \nabla_{F}(\mathbf{s})\right)
\end{aligned}
$$

\section{EXPERIMENTS AND CONCLUSION}

Figure 4 presents our experiments for Itakura-Saito and Kullback-Leibler divergences. Empirical studies suggest that the theoretical convergence is well below the upper bound $\frac{1}{T}$ of [3]. Figure 5 depicts the observed convergence rate for the Kullback-Leibler divergence. Banerjee et al. [2] proved that $\operatorname{Min} \operatorname{Avg}\left(D_{F}\right)$ is always the centroid whatever the Bregman divergence, and described a bijection between Bregman divergences and the exponential families in statistics. We exhibit yet another bijection between Bregman divergences and functional averages of core-sets [4].

\section{REFERENCES}

[1] F. Nielsen and R. Nock. Approximating smallest enclosing balls. In ICCSA CGA, pp. 147-157. LNCS \#3045, Springer-Verlag, pp. 147-157, 2004.

[2] A. Banerjee, S. Merugu, I. S. Dhillon, and J. Ghosh. Clustering with Bregman divergences. In Journal of Machine Learning (JMLR), 6:1705-1749, 2005.

[3] M. Bădoiu and K. L. Clarkson. Smaller core-sets for balls. In ACM-SIAM Sympos. Discret Algorithms, pp. 801-802, 2003. http://cm.bell-labs.com/who/clarkson/

[4] R. Nock and F. Nielsen. Fitting the smallest enclosing Bregman ball. In European Conference on Machine Learning (ECML), LNCS \#3720, pp. 649-656, 2005.

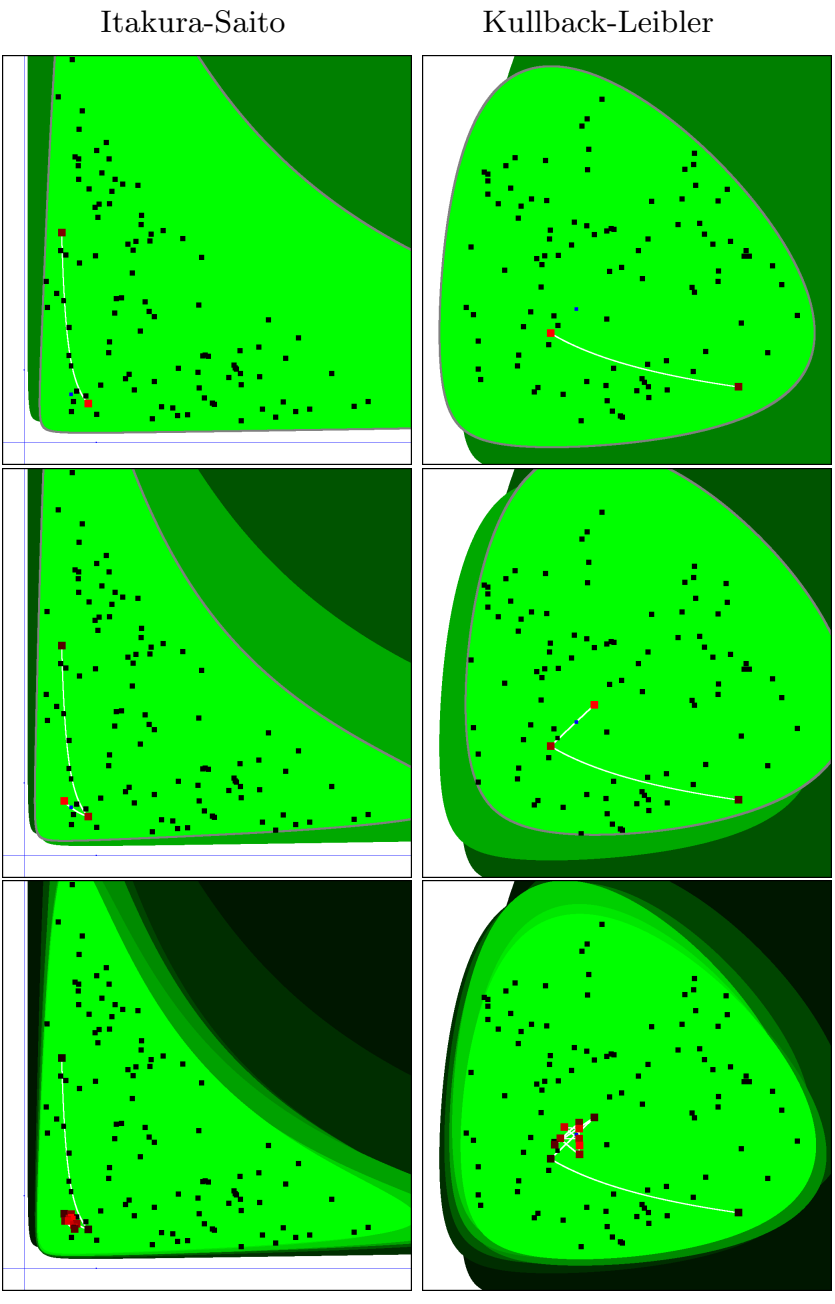

Figure 4: Algorithm BBC: Bregman enclosing balls after the first, second, and 10th iteration for the Itakura-Saito and Kullback-Leibler divergences. White lines depict the geodesics followed by c.

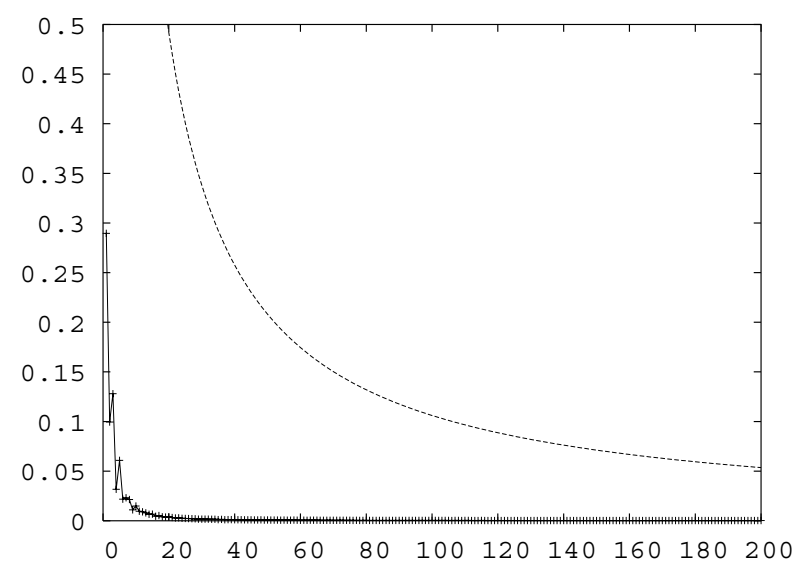

Figure 5: Convergence rate for the Kullback-Leibler divergence: $n=1000, d=2$ on 100 runs. Plain curve represent $\frac{D_{F}\left(\mathbf{c}^{*}, \mathbf{c}\right)+D_{F}\left(\mathbf{c}, \mathbf{c}^{*}\right)}{2}$, and dashed curve is the $\frac{1}{T}$ upperbound for $L_{2}^{2}[3]$. 